\title{
Metazoos parásitos de la mojarrilla Stellifer minor (Tschudi) (Osteichthyes, Sciaenidae) capturados por pesquería artesanal en Chorrillos, Lima, Perú
}

\author{
José lannacone \\ Laboratorio de Ecofisiología Animal, Facultad de Ciencias Naturales y Matemáticas, Universidad Nacional Federico Villarreal. \\ Calle San Marcos 383, Lima 21, Perú. E-mail: joseiannacone@hotmail.com
}

\begin{abstract}
Metazoan parasites of the minor stardrum, Stellifer minor (Tschudi) (Osteichthyes, Sciaenidae), caught by artisanal fishery on Chorrillos, Lima, Peru. A research on some community components of parasitefauna of 105 specimens of Stellifer minor (Tschudi, 1844) collected from Chorrillos Fishmarket, Lima, Peru, between May and October 1998 and necropsied to study parasite communities was conducted. Of the fishes collected, 71 were males and 34 females. Fishes showed a standard length between 10.20 and $20.50 \mathrm{~cm}$ (mean = $15.50 \pm 1.65$ ). Metazoan parasites were collected and counted employing conventional techniques. 3483 specimens in total during all the survey, with a mean abundance of 33.17 (3-122) were collected. The mean parasite species richness was 1.9 (1-4). One host was not parasited. Twenty hosts (19.04\%) showed infection with one parasite species, and seventy-seven (73.33\%) and seven (6.66\%) had multiple infection, two and three parasite species, respectively. Five parasites: Rhamnocercus oliveri Luque 8 lannacone, 1991 and $R$. stelliferi Luque $\&$ lannacone, 1991 (Monogenea) (prevalence $=98.09 \%$; mean intensity $=28.85 ;$ mean abundance $=28.58$ ), Clavellotis dilatata (Kroyer, 1863) (Copepoda) (prevalence $=2.85 \%$; mean intensity $=1$; mean abundance $=0.02$ ), Helicometra fasciata (Rudolphi, 1819) (Digenea) (prevalence $=79.04 \%$; mean intensity $=5.66$; mean abundance $=4.47$ ) and Procamallanus (Spirocamallanus) pereirai Annereaux, 1946 (Nematoda) (prevalence $=4.76 \%$; mean intensity $=1.6$; mean abundance $=0.07$ ) were found. Effect of the sex on mean intensity and abundance of Infection of Rhamnocercus Monaco, Wood \& Mizelle, 1954 and also effect of sex with mean abundance of infection with $H$. fasciata were found. The mean diversity in the infracommunities of $S$. minor was $\left(\mathrm{H}^{\prime}\right)=0.11$ and Simpson $\operatorname{Index}(C)=0.98$. Finally, the results of community assemblages with the parasite communities registered on $S$. minor ten years ago in the same locality of study were compared.
\end{abstract}

KEY WORDS. Clavellotis, Helicometra, parasite communities, parasites, Procamallanus, Rhamnocercus.

RESUMEN. Se investigaron algunos componentes comunitarios de la parasitofauna de 105 ejemplares de Stellifer minor (Tschudi, 1844) colectados del Terminal Pesquero de Chorrillos, Lima, Perú, entre el mayo y octubre de 1998 y necropsiados para estudiar sus comunidades parasitarias. De los peces colectados, 71 fueron machos y 34 hembras. Los peces mostraron una longitud estándar entre 10,20-20,50 cm (promedio $=15,50 \pm 1,65$ ). Los parásitos metazoos fueron colectados y censados empleando las técnicas convencionales. Se colectaron un total de 3483 especimenes durante todo el muestreo, con una abundancia media total de 33,17 (3-122). El promedio de la riqueza de especies de parásitos fue 1,9 (1-4). Un hospedero no presentó ningún parásito. 20 hospederos $(19,04 \%)$ mostraron infección con un solo parásito, 77 (73,33\%) y 7 (6,66\%) tuvieron una infección múltiple, con 2 y 3 especies de parásitos, respectivamente. Se encontraron cinco parásitos: Rhamnocercus oliveri Luque 8 lannacone, 1991 y R. stelliferi Luque \& lannacone, 1991 (Monogenea) (prevalencia = 98,09\%; intensidad media = 28,85; abundancia media = 28,58), Clavellotis dilatata (Kroyer, 1863 ) (Copepoda) (prevalencia = 2,85\%; intensidad media = 1; abundancia media = 0,02), Helicometra fasciata (Rudolphi, 1819) (Digenea) (prevalencia $=79,04 \%$; intensidad media $=5,77$; abundancia media $=4,56$ ) y Procamallanus (Spirocamallanus) pereirai Annereaux, 1946 (Nematoda) (prevalencia = 4,76\%; intensidad media = 1,6; abundancia media = 0,07). Se encontró efecto del sexo en la intensidad y abundancia media de Infección de Rhamnocercus Monaco, Wood \& Mizelle,1954 y también efecto del sexo con la abundancia media de infección con $H$. fasciata. La diversidad media de las infracomunidades según el índice de Shannon-Weaver de $S$. minor fue $\left(H^{\prime}\right)=0,11$ y el índice de Simpson $(C)=0,98$. Se compararon los resultados obtenidos con la estructura comunitaria parasitaria registrada en la década anterior en $S$. minor en la misma localidad.

PALABRAS CLAVE. Clavellotis, comunidad parasitaria, Helicometra, parásitos, Procamallanus, Rhamnocercus. 
Stellifer minor (Tschudi, 1844) “Mojarrilla”, distribuido en las costas de Perú y Chile (Chirichigno \& Velez 1998), es un pez carnívoro, bentófago y eurífago de importancia en los fondos arenosos marinos donde se alimenta preferentemente de invertebrados y algas verdes laminares (Ulva) (TARAZONA et al. 1988).

Se han descrito y registrados veintiún parásitos metazoos para S. minor en el Perú (Luque \& I annacone 1989 1991, Oliva et al. 1989, 1990, Luque et al. 1991, TANTALÉAN \& Huiza 1994, Luque 1996, JARA 1998).

Oliva et al. (1990), Luque (1996) y Oliva \& Luque (1998) han realizado una primera aproximación cuantitativa de los parásitos de S. minor entre el periodo de septiembre de 1987 a agosto de 1988 en el Terminal Pesquero de Chorrillos, Lima, Perú. Posteriormente no se ha efectuado ningún análisis cuantitativo de las comunidades parasitarias en estehospedero en esta misma localidad.

En Latinoamérica, el Niño 1997-98, conocido también como la alteración Sureña El Niño ha producido enormes cambios en el ámbito de especies, poblaciones y comunidades, tanto marinas como terrestres (IANNACONE et al. 2000, IANNACONE $\&$ AYALA 2004). En el ambiente marino se producen variaciones en la temperatura y en la salinidad, aparecen otras especies y la abundancia de un determinado recurso aumenta cuando otro disminuye. Estos cambios implican un "reacomodo" ecológico (Gutierrez 2001). En el caso de S. minor, amplió su distribución y abundancia en la costa peruana duranteel Niño 1997-98 (KameYA et al. 2001).

Este trabajo representa un análisis cuantitativo de las comunidades parasitarias de S. minor durante 1998 con el objetivo de evaluar la influencia del tamaño y sexo del hospedero, sobre las principales especies componentes de su fauna parasitaria.

\section{MATERIALY MÉTODOS}

Se adquirieron 105 especímenes de “Mojarrilla”, S. minor entre mayo y octubre de 1998 en el Terminal Pesquero de Chorrillos - Lima, Perú (12³0'S, 76 50'W). Solo branquias, cavidad bucal, estómago e intestino fueron examinados para la búsqueda de parásitos. Los parásitos se colectaron, fijaron, preservaron, colorearon y montaron siguiendo las recomendaciones de EIRAS et al. (2000) y IANNACONE et al. (2001).

Especimenes representativos de los monogeneos Rhamnocercus oliveri Luque \& Iannacone, 1991 y Rhamnocercus stelliferi Luque \& Iannacone, 1991; y del trematode Helicometra fasciata (Rudolphi, 1819) fueron depositados en la colección helmintológica del Museo de Historia Natural de la Universidad Nacional Mayor de San Marcos (MUSM-UNMSM), MUSM ${ }^{\circ} 1727$, MUSM $^{\circ} 1728$, y MUSM $^{\circ} 1726$, respectivamente. Especimenes del copépodo $C$. dilatata fueron depositados en la colección de invertebrados menores del mismo museo (CIR-UNMSM), CIR 252. Especímenes representativos de Procamallanus (Spirocamallanus) pereirai Annereaux, 1946 fueron depositados en la hel mintoteca de la Facultad de Ciencias Naturales y Matemáticas de la Universidad Nacional Federico Villarreal, Lima.

Se determinó en los hospederos, el sexo y la longitud estándar (en cm). La longitud estándar de los hospederos se dividió en seis rangos de 1,7 cm cada uno aplicando la regla de Sturges (ZAR 1996). Estos rangos fueron: 10,2-11,9 cm $(\mathrm{n}=4)$; $12,0-13,6 \mathrm{~cm}(\mathrm{n}=8) ; 13,7-15,3 \mathrm{~cm}(\mathrm{n}=36) ; 15,4-17,0 \mathrm{~cm}(\mathrm{n}=$ $45) ; 17,1-18,7 \mathrm{~cm}(\mathrm{n}=9)$ y $18,8-20,5 \mathrm{~cm}(\mathrm{n}=3)$. Los machos presentaron una longitud de $15,54+1,63 \mathrm{~cm}, \mathrm{n}=71$. Las hembras presentaron una longitud de $15,42 \pm 1,72 \mathrm{~cm}, \mathrm{n}=34$.

Se determinó la prevalencia, intensidad media y abundancia media por pez para todos los parásitos encontrados. Para las dos especies de Rhamnocercus (R. stelliferi y R. oliveri) el análisis cuantitativo fue realizado en conjunto a. El Indice de dispersión (ID) empleado, se determinó de la relación entre Varianza (S2) / intensidad media y evaluado medianteel estadístico d. El análisis de los metazoos parásitos a nivel de infracomunidades y comunidades parasitarias se hizo para las especies con prevalencias mayores al 10\%, de acuerdo a Esch et al. (1990).

La prueba det de Student, previa evaluación de homogeneidad de varianzas empleando la prueba de Levene, fue usada para determinar si la longitud estándar de los peces hospederos machos y hembras presentaban diferencias significativas. La influencia de la talla del hospedero en la prevalencia de infección de los parásitos se determinó usando el coeficiente de correlación de Pearson ( $r$ ). El coeficiente de correlación deSpearman se usó para determinar la relación del tamaño del hospedero con la intensidad media y la abundancia media para cada parásito. Se aplicó la prueba $\chi^{2}$ para tablas de contingencia para determinar el grado de dependencia entreel sexo del hospedero y la prevalencia parasitaria. El efecto del sexo en la intensidad media y la abundancia media de infección parasitaria se calculó utilizando la prueba de te Student (ZAR 1996).

La diversidad parasitaria de cada infracomunidad fue calculada a través del índice de Shannon Weaver $\left(\mathrm{H}^{\prime}\right)$ y del índice de dominancia de Simpson (IANNACONE et al. 2003). El nivel de significancia fue evaluado a a =0,05. La terminología ecológica (prevalencia, abundancia e intensidad media) siguió los criterios de BusH et al. (1997). Para el cál culo de las pruebas estadísticas descriptivas e inferenciales se usó el paquete estadístico SPSS 7,5 para Windows 95.

\section{RESULTADOS}

La tabla I muestra la prevalencia, intensidad y abundancia media de infestación de los parásitos encontrados en los 105 hospederos muestreados de $\mathrm{S}$. minor. Además en los parásitos más prevalentes como R. oliveri $+\mathrm{R}$. stelliferi, y $\mathrm{H}$. fasciata se observó una distribución sobredispersa (distribución binomial negativa), pues el Índice de dispersión fue mayor a 1 y el estadístico d fue de 43,41 y de 15,6, respectivamente. La mayor frecuencia de dominancia fue para R. oliveri $+R$. stelliferi (Tab. II). Para el caso de P. pereirai el estadístico d fue de -8,26, lo que indicó una dispersión uniforme. 
Tabla I. Prevalencia, intensidad y abundancia de los parásitos de Stellifer minor en el terminal pesquero de Chorrillos, Lima, Perú. (ND) No determinado.

\begin{tabular}{|c|c|c|c|c|}
\hline Parásito & $\begin{array}{l}\text { Prevalencia } \\
\text { (\%) }\end{array}$ & Intensidad Media (Rango) & $\begin{array}{l}\text { Abundancia Media } \\
\text { (Rango) }\end{array}$ & Índice de Dispersión \\
\hline \multicolumn{5}{|l|}{ Monogenea } \\
\hline Rhamnocercus oliveri + Rhamnocercus stelliferi & 98,09 & $28,85 \pm 21,53(1-122)$ & $28,58 \pm 21,61(0-122)$ & 16,06 \\
\hline \multicolumn{5}{|l|}{ Trematoda } \\
\hline Helicometra fasciata & 79,04 & $5,66 \pm 4,94(1-20)$ & $4,47 \pm 4,94(0-20)$ & 4,31 \\
\hline \multicolumn{5}{|l|}{ Nematoda } \\
\hline Procamallanus (Spirocamallanus) pereirai & 4,76 & $1,60 \pm 0,54(1-2)$ & $0,07 \pm 0,35(0-2)$ & 0,18 \\
\hline \multicolumn{5}{|l|}{ Copepoda } \\
\hline Clavellotis dilatata & 2,85 & $1 \pm 0(1)$ & $0,02 \pm 0,16(0-1)$ & ND \\
\hline
\end{tabular}

Tabla II. Frecuencia de dominancia de dos parásitos componentes de Stellifer minor de la Costa Central en Chorrillos, Lima- Perú.

\begin{tabular}{lcc}
\hline \multicolumn{1}{c}{ Parásito } & Frecuencia de Dominancia & Frecuencia de Dominancia de dos especies \\
\hline Rhamnocercus oliveri + Rhamnocercus stelliferi & 89 & 1 \\
Helicometra fasciata & 14 & 1 \\
\hline
\end{tabular}

El promedio de la longitud de los peces machos $(15,54$ $\pm 1,63 \mathrm{~cm})$ y de las hembras $(15,42 \pm 1,72 \mathrm{~cm})$ asumiendo igual dad de varianzas no fue significativamente diferente $(\mathrm{t}=$ $0,32 ;$ g.l. $=103 ; p=0,74)$.

La tabla III indica la ausencia de relación lineal con la talla del hospedero y la prevalencia, intensidad y abundancia media de R. oliveri + R. stelliferi y $\mathrm{H}$. fasciata. Solo se encontró efecto entre el sexo y la intensidad (Machos $=25,58 \pm 20,81$; Hembras $=35,58 \pm 21,73$ ), y abundancia media de infestación (Machos =25,22 $\pm 20,88$; Hembras $=35,58 \pm 21,73$ ), respectivamente para Rhamnocercus; así como efecto del sexo y la abundancia media de infección de H. fasciata (Machos $=3,74 \pm$ 4,43; Hembras $=6,00 \pm 5,65)$ (Tab. IV).

Ciento cuatro $(99,04 \%)$ mojarrillas estuvieron parasitadas por lo menos con una especie de parásito. Se colectaron un total de 3483 especímenes de parásitos durante todo el muestreo, con una abundancia media total de 33,17 (rango = 3-122) y con una riqueza de especies de parásitos de 1,9 (rango $=1-4$ ), Ias que no estuvieron correlacionadas con la longitud total corporal $(p>0,05)$. Sesenta y dos hospederos mostraron más de tres especímenes por pez.

Un hospedero no presentó ningún parásito. El monoparasitismo se encontró en 20 hospederos (19,04\%), el biparasitismo en 77 hospederos (73,33\%) y el triparasitismo en $7(6,66 \%)$ hospederos. La diversidad media de las infracomunidades ectoparasitarias de $\mathrm{S}$. minor fue $\mathrm{H}^{\prime}=0,11$ y el índice de Simpson $(C)=0,98$.

La tabla $\mathrm{V}$ señala un análisis comparativo entre los parámetros ecológicos de R. oliveri $+\mathrm{R}$. stelliferi y de H. fasciata entre septiembre 1987 - agosto 1988 y mayo-octubre 1998 (presente estudio), observándose una disminución en la intensidad media de 165,4 a 28,85 para Rhamnocercus y de 13,6 a 5,66 para $H$. fasciata. En ambos casos la prevalencia de infección se mantiene con ligera variación (Tab. V).

\section{DISCUSIÓN}

Helicometra fasciata $(=\mathrm{H}$. pulchella) ha sido registrada a Io largo de la Costa Peruana en nueve hospederos diferentes (Luque et al. 1991, JaRA 1998, Oliva \& Luque 1998, Oliva \& Luque 2002). CRIBB et al. (2002) consideran a Opecoelidae, que incluye a H. fasciata, como digeneos dominantes en peces teleosteos carnívoros, lo cual explicaría la alta dispersión de esta especie, por encontrarse en familias de peces teleosteos en el Perú, que adquieren la metacercaria de un amplio rango de invertebrados, aún desconocidos para el Perú.

OlIVA \& LUQUe (1998) señalan quela principal característica de las comunidades parasitarias en cinco peces marinos de la costa peruana, entre ellos S. minor, es la fuerte predominancia de monogeneos ectoparásitos. Este mismo patrón se ha encontrado durante 1998 con especies del genero del monogeneo Rhamnocercus Monaco, Wood \& Mizelle, 1954 parasitando a S. minor, al presentar la más alta dominancia (Tab. II).

RoHde et al. (1995) señal an que la abundancia, la riqueza de especies de parásitos fue significativamente correl acionada con la longitud del hospedero, en un análisis realizado en 102 especies de peces marinos. Oliva et al. (1990) encontraron que R. oliveri + R. stelliferi y $\mathrm{H}$. fasciata en S. minor durante 19871988, no presentaron relación entre la longitud del pez y la prevalencia de infección. Esta ausencia de relación fue encontrada en el presente trabajo (Tab. III). OLIVA et al. (1990) encontraron quela Intensidad media de ambos parásitos estuvo relacionado linealmente con la longitud de $\mathrm{S}$. minor durante 
Tabla III. Valores de los coeficientes de correlación de Spearman (rs) y del coeficiente de correlación ( $r$ ) usados para evaluar la relación posible entre la longitud estándar de Stellifer minor y la abundancia, intensidad y prevalencia de dos de sus parásitos componentes de su comunidad parasitaria en la Costa Central de Lima, Perú. (p) Nivel de significancia, (rs) coeficiente de correlación de Spearman, (*) longitud estándar vs abundancia media, (**) longitud estándar vs intensidad media, (***) longitud estándar vs prevalencia.

\begin{tabular}{lcccccc}
\hline \multicolumn{1}{c}{ Parásito } & $\mathrm{rs} *$ & $\mathrm{p}$ & $\mathrm{rs} * *$ & $\mathrm{p}$ & $\mathrm{r} * * *$ & $\mathrm{p}$ \\
\hline Rhamnocercus oliveri +Rhamnocercus stelliferi & 0,12 & 0,81 & 0,03 & 0,73 & 0,05 & 0,61 \\
Helicometra fasciata & 0,08 & 0,97 & 0,08 & 0,44 & 0,05 & 0,56 \\
\hline
\end{tabular}

Tabla IV. Valores de la prueba de t de Student y del estadístico $\chi^{2}$ usados para evaluar la posible relación entre el sexo de Stellifer minor y la intensidad, abundancia y prevalencia de dos de sus parásitos componentes de su comunidad parasitaria en la Costa Central de Lima, Perú ( $p=$ Nivel de significancia). (*) Intensidad media; (**) abundancia media.

\begin{tabular}{lcccccc}
\hline \multicolumn{1}{c}{ Parásito } & $\mathrm{t}^{*}$ & $\mathrm{p}$ & $\mathrm{t}^{* *}$ & $\mathrm{p}$ & $\chi^{2}$ \\
\hline Rhamnocercus oliveri +Rhamnocercus stelliferi & 2,26 & 0,02 & 2,34 & 0,02 & 0,14 & 0,70 \\
Helicometra fasciata & 1,24 & 0,21 & 2,04 & 0,04 & 3,44 & 0,06 \\
\hline
\end{tabular}

Tabla V. Análisis comparativo cuantitativo de dos componentes de la fauna parasitaria de Stellifer minor entre 1987-88 y 1998. (ID) Índice de dispersión.

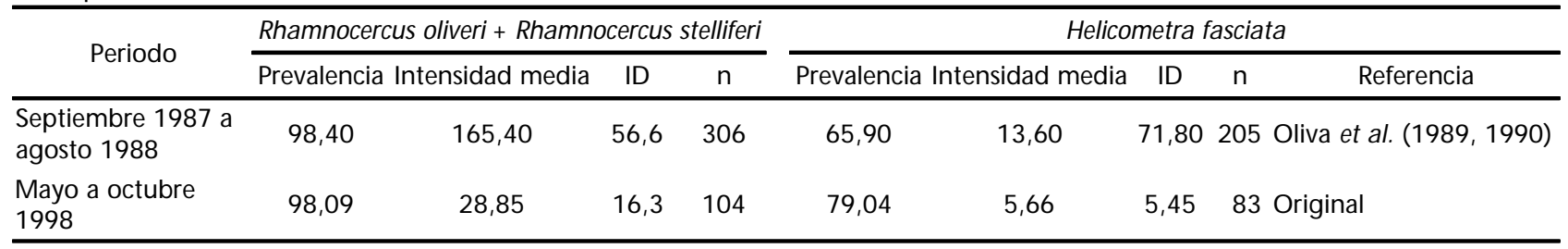

1987-88. Esta relación lineal no fue encontrada en el presente estudio (Tab. III).

La dependencia encontrada entre el sexo y la Intensidad media de las dos especies de Rhamnocercus, ha sido encontrada en nuestro estudio (Tab. IV) y en el de OLIVA et al. (1990). En S. minor se encontró que los machos presentaban mayor abundancia media de infección que las hembras para R. oliveri + R. stelliferi y H. fasciata. I Annacone (2003) indican que la preferencia a uno de los sexos de los hospederos pudiera ser atribuida a la diferencia en las relaciones ecológicas (comportamiento, hábitat y dieta) de los peces machos y hembras.

JARA (1998) registró en la zona norte del mar peruano, en el Terminal Pesquero deTrujillo en 100 especímenes deS. minor entre agosto y octubre de 1995, a siete especies de helmintos parásitos: cuatro monogeneos (Encotyllabe sp., P. bravoi, Loimos sp. y Rhamnocercus sp.), un digeneo $\mathrm{H}$. fasciata, un nemátodo Anisakis sp. y un acantocéfalo Corynosoma obtuscens Lincicome, 1943. De los cuales solo Rhamnocercus sp. y H. fasciata fueron coincidentes en el presente estudio, y remarca la alta variabilidad espacial y temporal de la fauna parasitaria de S. minor.

Oliva et al. (1990) encontraron a dos monogeneos: Pedocotyle bravoi Luque \& Iannacone, 1989 y Pedocotyle annakohni Luque \& Iannacone, 1989 como especies con prevalencias entre $14,5 \%$ y $30,5 \%$ durante $1987-88$, los cuales estuvieron ausentes durante 1998.

El presente estudio se inició al final de El Niño 19971998 (que ocasionó anomalías térmicas entre febrero 1997 a julio 1998 entre 3 a $8^{\circ} \mathrm{C}$ en la costa central del Perú) (MORON \& SARMIENTO 2001). Muchos organismos marinos son muy sensibles a El Niño en su frecuencia de ocurrencia en dependencia delas fluctuaciones espacio-temporales. Algunas especies ícticas tienden a incrementarse durante El Niño y el post-Niño (Bouchon et al. 2001). KAMEYA et al. (2001) consideraran a S. minor como una especie bioindicadora de aguas ecuatoriales cálidas durante El Niño 1997-98, debido a que incrementó su distribución y abundancia hacia el sur del Perú.

OlIVA \& LUQUe (1998) indican queel fenómeno deEl Niño, alteró dramaticamente la estructura parasitaria de cinco peces esciénidos de Perú. Tarazona et al. (2001) consideran que las especies indicadoras de El Niño pueden ser de dos tipos: las que producen cambios en su distribución (presencia o ausencia temprana o tardía de una especie durante El Niño), posiblemente este patrón podría aplicar a los monogeneos $\mathrm{P}$. bravoi y P. annakohni, que estuvieron ausentes durante el presente estudio (Tab. I); y las que lo indican con sus cambios en su abundancia, como posiblemente serían las especies de Rhamnocercus y $\mathrm{H}$. fasciata (Tab. V). Es necesario mencionar que el primer estudio (1987-1988) incluyó un análisis ecológi- 
co de doce meses y este segundo solo de siete meses (parte de otoño, invierno y parte de primavera).

En el presente estudio solo censamos a cinco especies de parásitos: R. oliveri +R. stelliferi (filamento branquial), C. dilatata (rastrillo branquial), H. fasciata (Intestino y ciegos intestinales) y P. pereirai (Intestino).

\section{AGRADECIMIENTOS}

El autor expresa su agradecimiento al Laboratorio de Ecofisiología Animal de la Facultad de Ciencias Naturales y Matemáticas de la Universidad Nacional Federico Villarreal por su apoyo en la presente investigación.

\section{REFEREN CIA S BIBLIO GRÁ FICAS}

Bouchon, M.; S. Cahuín \& M. Ñ IQuen. 2001. Fluctuaciones de la ictiofauna pelágica en la región norte-centro del litoral peruano de 1994 a 1998, p. 73-79. In: J. Tarazona; W. Arntz \& E. CASTillo (Eds). El Niño en América Latina. Impactos biológicos y sociales. Lima, Consejo Nacional de Ciencia y Tecnología, Ed. Omega, 423p.

Bush, A.O.; K.D. Lafferty; J.L. Lotz \& A.W. Shostak. 1997. Parasitology meets ecology on its own terms: Margolis et al. revisited. Journal of Parasitology, Washington, D.C., 83: 575-583.

Chirichigno, N. \& M. Velez. 1998. Clave para identificar los peces marinos del Perú. Callao, Ed. Callao, Publicación Especial del Instituto del Mar, $2^{\text {da }}$, 500p.

CribB, T.H.; L.A. Chistholm \& R.A. Bray. 2002. Diversity in the Monogenean and Digenea: does lifestyle matter? I nternational Journal for Parasitology, Amsterdam, 32: 321-328.

EIRAS, J.; R. TAKemoto \& G.C. PavanelLI. 2000. Métodos de estudo e técnicas laboratoriais em parasitología de peixes. Maringá, Editora Universidade Estadual de Maringá, 171p.

Esch, G.W.; A.W. ShostaK; D.J. Marcogliese \& T.M. Goater. 1990. Patterns and process in helminth parasite communities: an overview, p. 1-19. In: G. Esch; A.C. Bush \& J. Ано (Eds). Parasite Communities: Patterns and processes. New York, Chapman and Hall, 251p.

GutierReZ, M. 2001. Efecto del evento El Niño 1997-98 sobre la distribución y abundancia de anchoveta (Engraulis ringens), p. 55-72. In:J. Tarazona; W. Arntz \& E. Castillo (Eds). El Niño en América Latina. Impactos biológicos y sociales. Lima, Consejo Nacional de Ciencia y Tecnología, Ed. Omega, 423p.

IANNACONE, J. 2003. Tres metazoos parásitos de la cojinoba Seriolella violacea Guichenot (Pisces, Centrolophidae), Callao, Perú. Revista Brasileira de Zoología, Curitiba, 20: 257-260.

Iannacone, J. \& L. Ayala. 2004. Censo de Ornithodoros amblus Chamberlin (Acarina: Argasidae) en Ia isla Mazorca, Lima, Perú. Parasitología Latinoamericana, Santiago de Chile, 59: 56-60.

Iannacone, J.; L. Alvariño; R. Moreno; M. Reyes \& J. Chauca. 2000.
Culícidos (Diptera) del río Chillón y sectores adyacentes de la Provincia constitucional del Callao, Perú, duranteEl Niño 1997-98. Acta Entomológica Chilena, Santiago de Chile, 24: 51-60.

Iannacone, J.; J. Tataje; J. Fuentes-Rivera; K. Álvarez \& P. Aguilar. 2001. Infracomunidades ectoparasitarias en las branquias de la cachema Cynoscion analis Jenyns (Pisces: Sciaenidae). Revista Peruana de Parasitología, Lima, 15: 42-54.

Iannacone, J.; L. Alvariño; A. Guabloche; M. Alayo; J. Sánchez; A. Arrascue \& M. Abanto. 2003. Comunidades ectoparasitarias branquiales de la pintadilla Cheilodactylus variegatus Valenciennes 1833 (Pisces: Cheilodactylidae). Parasitología Latinoamericana, Santiago de Chile, 58: 59-67.

JARA, C. 1998. Prevalencia e intensidad de parasitismo por helmintos en cuatro especies de peces de la zona norte del mar peruano. Revista Peruana de Parasitología, Lima, 13: 7683.

Kameya, A.; M. Lleellish \& L. Caccha. 2001. Los peces como indicadores de El Niño en el ecosistema marino peruano desde 1972 a 1998, p. 81-89. In: J. Tarazona; W. Arntz \& E. CASTILLO (Eds). EI Niño en América Latina. Impactos biológicos y sociales. Lima, Consejo Nacional de Ciencia y Tecnología, Ed. Omega, 423p.

Luque, J.L. 1996. Distribución transversal y asociaciones interespecífica en las comunidades de metazoarios ectoparásitos de peces esciénidos marinos del Perú. Revista de Biología Tropical, San José de Costa Rica, 44: 383.390.

Luque, J.L. \& J. I Annacone. 1989. Pedocotyleannakohni sp. nov. y Pecodotyle bravoi sp. nov. (Monogenea: Diclidophoridae) parásitos de Stellifer minor T. (Teleostei: Sciaenidae) en la costa peruana. Revista I bérica de Parasitología, Madrid, 49: 301-306.

. 1991. Rhamnocercidae (Monogenea: Dactylogyroidea) in Sciaenid fishes from Perú, with description of Rhamnocercoides menticirrhi n. gen. sp. nov. and two species of Rhamnocercus. Revista de Biología Tropical, San José de Costa Rica, 39: 193-201.

Luque, J.L.; J. Iannacone \& C. Farfán. 1991. Parásitos de peces óseos marinos en el Perú: Lista de especies conocidas. Boletín de Lima 74: 17-28.

Moron, O. \& M. SARMiento. 2001. Aspectos oceanográficos del El Niño 1997-98 y su relación con los recursos pelágicos, p. 5-27. In: J. Tarazona; W. Arntz \& E. Castillo (Eds). El Niño en América Latina. Impactos biológicos y sociales. Lima, Consejo Nacional de Ciencia y Tecnología, Ed. Omega, 423p.

Oliva, M.E. \& J.L. Luque. 1998. Metazoan parasites infracommunities in five sciaenids from the Central Peruvian Coast. Memorias do Instituto Oswal do Cruz, Rio de Janeiro, 93: 175-180.

. 2002. Endohelminth parasites of the Trambollo Labrisomus philippii (Steindachner) (Osteichthyes: Labrisomidae) from the Central Peruvian Coast. Comparative Parasitology, Washington, D.C., 69: 100-104. 
Oliva, M.E.; J.L. Luque \& J. IAnnacone. 1989. Prevalencia y patrones de distribución de tres especies de monogeneos parásitos de Stellifer minor (Tschudi, 1844). Revista Ibérica de Parasitología, Madrid, 49: 209-214.

. 1990. The metazoan parasites of Stellifer minor (Tschudi, 1844): An ecological approach. Memorias do Instituto Oswaldo Cruz, Rio de Janeiro, 85: 271-274.

Rohde, K.; C. HaYward \& M. Heap. 1995. Aspects of the ecology of metazoan ectoparasites of marine fishes. International Journal for Parasitology, Amsterdam, 25: 945-970.

Tantaleán, M. \& A. Huiza. 1994. Sinopsis de los parásitos de peces marinos de la costa peruana. Biotempo (Perú), Lima, 1: 53-101.

Tarazona, J.; W. Arntz \& L. Hoyos. 1988. Repartición de los re-

Recebido em 02.III.2004; aceito em 22.X.2004. cursos alimenticios entretres peces bentófagos frenteal Perú antes, durante y después de El Niño 1982-83, p. 107-114. In: H. Salzwedel \& A. LANDA (Eds). Recursos y dinámica del ecosistema de afloramiento peruano. Memorias del $2^{\text {do }}$ Congreso Latinoamericano sobre Ciencias del Mar (COLACMAR), 17-21 Agosto de 1987. Tomo I. Instituto del $M$ ar del Perú. Boletín volumen extraordinario Callao-Perú.

Tarazona, J.; W. Arntz; S. Valle \& T. Peña. 2001. Los índices de El Niño y del impacto sobre las comunidades bentónicas, $p$. 113-125. In: J. Tarazona; W. Arntz \& E. Castillo (Eds). El Niño en América Latina. Impactos biológicos y sociales. Lima, Consejo Nacional deCiencia y Tecnología, Ed. Omega, 423p.

ZAR, J.H. 1996. Biostatistical Analysis. New Jersey, PrenticeHall, Upper Saddle River, $3^{\text {th }}$ ed., 662p.

Revista Brasileira de Zoologia 21(4): 815-820, dezembro 2004 